\title{
Enhancing the Anticancer Effects of 5-Fluorouracil: Current Challenges and Future Perspectives
}

\author{
François Ghiringhelli ${ }^{1,2,3}$, Lionel Apetoh ${ }^{1,2,3}$
}

\begin{abstract}
5-Flurouracil (5-FU), a pyrimidine analog, was originally designed to prevent tumor cell growth. However, since the identification of its tumor inhibitory activity in 1957, substantial evidence has demonstrated that 5-FU could also harness the host immune system to prevent cancer progression. 5-FU sensitizes tumor cells to Natural Killer (NK) and CD8 T cell-driven cytotoxicity. We have also recently shown that 5-FU could selectively eliminate Myeloid Derived Suppressor Cells (MDSCs), which accumulate during cancer progression and compromise anticancer immune responses. The ability of 5-FU to trigger direct tumor cell death, enhance immune effector cell activation and eliminate immunosuppressive MDSCs explains its capacity to relieve tumor-induced immunosuppression and restore anticancer immune responses. Combination therapies

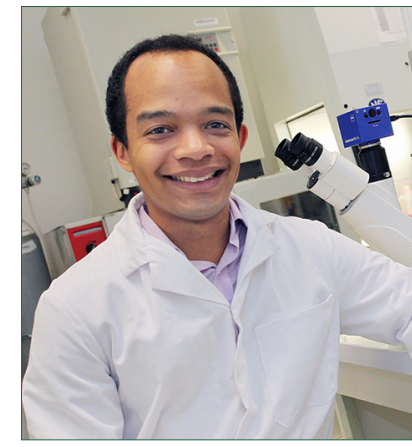

Dr. Lionel Apetoh using 5-FU with other chemotherapeutic agents, immunomodulators, or vaccines have further enhanced the clinical benefit of 5-FU. Here, we discuss how the increased understanding of the immune-driven effects of 5-FU prompts the design of relevant cancer chemoimmunotherapy strategies. (Biomed J 2015;38:111-116)
\end{abstract}

\section{Key words: 5-fluorouracil, anticancer immunity, immunomodulation, immunosuppression, inflammasome, myeloid-derived suppressor cell}

$\mathrm{T}_{1}$ The fluoropyrimidine 5-fluorouracil (5-FU) is an antimetabolite drug that prevents DNA and RNA synthesis and thus drives cell death. The rationale to design and use 5-FU as an anticancer agent stemmed from results obtained by Rutman et al. in $1954 .{ }^{[1]}$ They had previously noted that administration of uracil to rats receiving the carcinogen 2-acetylaminofluorene increased hepatoma formation. Following up on this observation, they found using radioactive-labeled uracil that this pyrimidine was used preferentially for nucleic acid biosynthesis in rat hepatoma compared to normal rat liver. ${ }^{[1]}$ These observations suggested that targeting the uracil metabolism would be effective in triggering tumor cell death. Heidelberger subsequently synthetized 5-fluoropyrimidine derivatives and unraveled the anticancer activity of the antimetabolite 5-FU. ${ }^{[2]}$ The mechanisms explaining the direct cytotoxic effects of 5-FU have been studied. 5-FU first enters cancer cells in a comparable manner to uracil and is transformed into the active metabolites such as fluorodeoxyuridine monophosphate, fluorodeoxyuridine triphosphate, and fluorouridine triphosphate. ${ }^{[3]}$ This series of events competitively blocks the enzymatic activity of thymidylate synthase, which is involved in the synthesis of thymine nucleotides and inhibits DNA synthesis. The cytotoxicity of 5-FU also relies on the ability of its metabolites to incorporate into DNA and RNA.

5-FU is still currently widely used for the treatment of breast and digestive cancers. It is routinely combined with other chemotherapies such as irinotecan and oxaliplatin to treat metastatic colon cancer as 5-FU-based combination therapies such as FOLFOX, FOLFIRI, or FOLFIRINOX (5-FU, oxaliplatin, irinotecan, and leucovorin), which could substantially improve patients' survival compared to monotherapy ${ }^{[4]}$ FOLFIRINOX therapy has also recently demonstrated superior anticancer efficacy compared to gemcitabine $(\mathrm{Gem})$ in metastatic pancreatic cancer patients. ${ }^{[5]}$ Nevertheless, a substantial research effort is still needed to

From the ${ }^{1}$ INSERM, U866, Dijon, France; ${ }^{2}$ Centre Georges François Leclerc, Dijon, France; ${ }^{3}$ Université de Bourgogne, Dijon, France Received: Jan. 28, 2014; Accepted: Apr. 09, 2014

Correspondence to: Dr. Lionel Apetoh, INSERM UMR866, Faculties of Medicine and Pharmacy, Dijon, France. 7 Bd Jeanne d'Arc, 21079 Dijon, France. Tel: 33-3-80393371; Fax: 33-3-80393434; E-mail: lionel.apetoh@inserm.fr

DOI: $10.4103 / 2319-4170.130923$ 
enhance the patients' response rate to 5-FU-based chemotherapy. Over the last 10 years, we and others have shown that anticancer immune responses are essential for the success of some chemotherapies in mice and humans. ${ }^{[6-10]} \mathrm{In}$ this regard, it has become clear that 5-FU has an ability to profoundly affect the host's immune system and anticancer immune responses, which have been shown to make a crucial contribution to the efficacy of some anticancer chemotherapies such as doxorubicin or oxaliplatin. 5-FU was shown to sensitize cancer cells to killing by effector CD8 T cells and to reduce the frequency of immunosuppressive myeloid-derived suppressor cells (MDSCs), suggesting that 5-FU could be used to design successful chemoimmunotherapeutic strategies. However, we have recently shown that 5 -FU treatment could trigger the release of interleukin (IL)- $1 \beta$ from MDSCs, thereby favoring tumor progression. ${ }^{[1]}$ Thus, understanding the immune-mediated effects of 5-FU is necessary to design effective combination therapies with 5-FU and immunomodulation. Here, we discuss how the knowledge gained from preclinical studies on the immune-based effects of 5-FU can be translated into successful therapeutic strategies combining chemotherapy and immunomodulation.

\section{5-FU activates immune effectors and eliminates immunosuppressive cells}

Contrary to the presiding view that chemotherapeutic agents trigger immunosuppression, we and others have shown that several anticancer drugs could instead promote the activation of immune effectors (reviewed in Refs ${ }^{[12,13]}$ ). 5-FU has also been shown to sensitize tumor cells to CD8 T-cell-dependent cytotoxicity [Figure 1]. Treatment of gastric cancer cells with 5-FU induced the expression of inducible
Heat Shock Protein 70 and favored tumor uptake by dendritic cells (DCs). ${ }^{[14]}$ In addition, DCs that had captured 5-FU-treated tumor cells featured enhanced IL-12 secretion and gastric carcinoma-associated antigen presentation capacity, resulting in increased interferon-gamma (IFN $\gamma$ ) release and cytotoxic activity from autologous CD8 T cells. ${ }^{[14]}$ Bergmann-Leitner and Abrams reached similar conclusions using human cancer colon cell lines. They found that 5-FU induced Intercellular Adhesion Molecule 1 (ICAM-1) and Fas expression in SW480 colon cancer cells, thereby driving their elimination through antigen-specific CD8 T cell-dependent killing. ${ }^{[15]} \mathrm{In}-$ terestingly, in colorectal cancer patients, 5-FU was also shown to induce Fas receptor expression, suggesting that 5-FU could trigger tumor cell death by promoting the engagement of the Fas/FasL pathway [Figure 1]. ${ }^{[16]}$ It has also been proposed that 5-FU could switch the polarization of peripheral blood mononuclear cells (PBMC) from healthy volunteers to a Th1 cytokine secretion profile, but the relevance of these results in patients remains to be determined..$^{[17]}$

While the aforementioned observations clearly indicated that 5-FU was able to promote immune activation, its effect on tumor-induced immunosuppression was elusive. Regulatory T cells (Tregs) and MDSCs are the major immunoregulatory cells involved in immune tolerance. ${ }^{[18,19]}$ These cells have been shown to expand in the blood, lymphoid organs, and in the tumor of cancer-bearing hosts. They have been shown to suppress both innate and adaptive immune responses and compromise anticancer immune responses. It has been already reported that conventional chemotherapies can target Tregs and MDSCs. Low-dose cyclophosphamide has been described to selectively target Tregs, thereby restoring natural killer (NK) cell and T cell activation in cancer patients. ${ }^{[20]}$ Similarly, Gem has been reported to

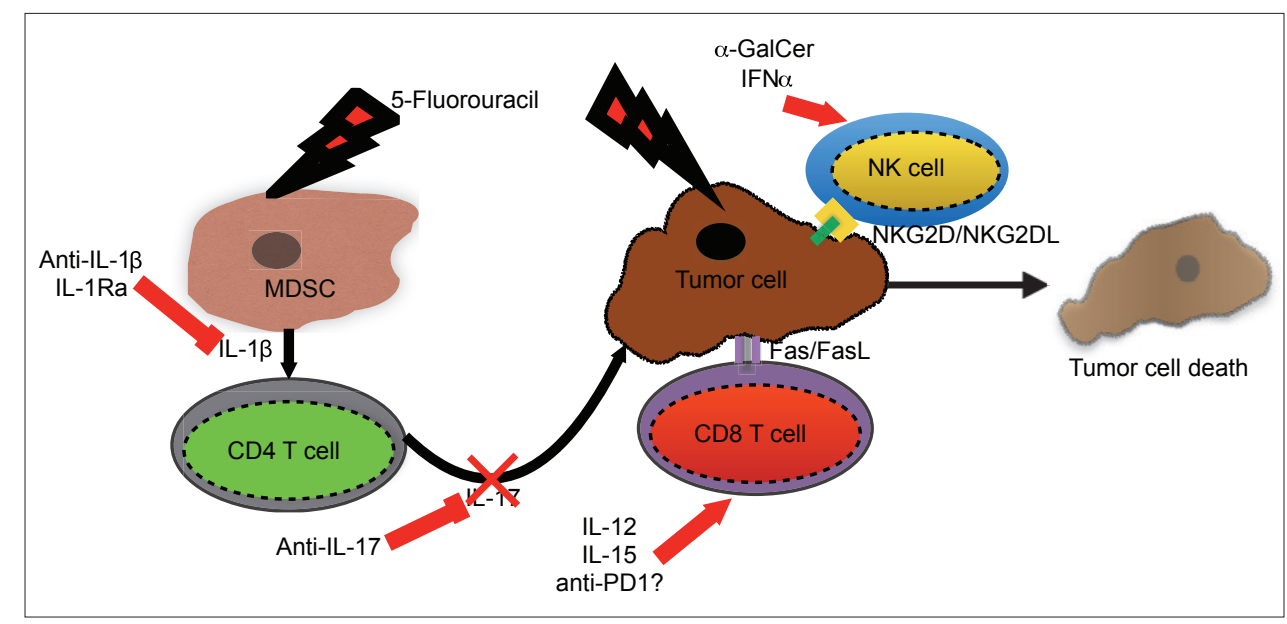

Figure 1: Therapeutic strategies designed to enhance 5-fluorouracil (5-FU) anticancer activity. 5-FU sensitizes the tumor cells to killing by NK and CD8 T cells through the upregulation of NKG2D ligands and Fas, respectively. 5-FU also eliminates MDSCs, thereby partially restoring CD8 T cell activation. However, 5-FU-induced MDSC cell death leads to release of IL-1 $\beta$, which enhances Th17 cell polarization, IL-17 secretion, and tumor angiogenesis. Combination therapies with 5-FU and immunomodulators such as IL-1Ra, anti-IL-17, or T-cell-activating cytokines could be beneficial to enhance 5-FU anticancer effects. 
eliminate MDSCs in mice. ${ }^{[21]}$ We have examined the ability of 5-FU to affect the biology of immunosuppressive cells in tumor-bearing mice. For this, we first administered 5-FU and Gem, paclitaxel, oxaliplatin, doxorubicin, and cyclophosphamide to mice bearing large EL4 thymoma tumors. Five days after treatment, we noted that only Gem and 5-FU reduced the frequency of circulating and tumor-infiltrating MDSCs. ${ }^{[22]}$ Additional immune monitoring revealed that 5-FU failed to alter the levels of circulating B, T, and NK cells, suggesting that the effect of 5-FU on MDSCs is selective. ${ }^{[22]}$ These findings were recently confirmed by Qu et al., who reported using a mouse model of B16 melanoma cancer cells growing intraperitoneally that Gem or 5-FU decreased MDSC numbers in the peritoneal cavity of tumor-bearing mice without altering the anticancer functions of mouse macrophages. ${ }^{[23]}$

We searched for the mechanism accounting for the activity of 5-FU on MDSCs and found that 5-FU directly triggered MDSC cell death in vitro and in vivo. By testing the presence of activated caspases in 5-FU-treated MDSC murine cell lines, we found that 5-FU drove MDSC apoptosis in vitro, as illustrated by the presence of activated caspases 3 and 7 in 5-FU-treated MDSCs. ${ }^{[22]}$ Similarly, caspase activation was detected in MDSCs, but not in other immune cells of 5-FU-treated tumor-bearing mice. In an attempt to explain the high sensitivity of MDSCs to 5-FU, we compared the expression levels of thymidylate synthase, the target enzyme of 5-FU metabolites as discussed above, between MDSCs, tumor cells, and splenocytes and noted that MDSCs exhibit low levels of this enzyme. ${ }^{[22]}$ This suggests that the selective apoptosis-inducing effect of 5-FU on MDSCs is due to their low expression of thymidylate synthase.

We and others have previously documented that oxaliplatin and doxorubicin could trigger cancer cell death that results in immune activation and the induction of anticancer immune responses. ${ }^{[6,7]}$ This concept of an immunogenic form of the cell death has been reviewed elsewhere. ${ }^{[24]}$ The first two signals that are required for the induction of immunogenic cell death are the cell surface expression of calreticulin, an endoplasmic reticulum protein driving tumor phagocytosis, and the cytoplasmic release of the nuclear protein high mobility group box 1 (HMGB1), which is responsible for the Toll-like receptor 4 (TLR4)-dependent cross-presentation of tumor antigens by DCs. ${ }^{[6,7]}$ Immunogenic chemotherapies have been proposed to "reset" the immune system and restore anticancer immunity. We tested whether the possible induction of immunogenic cell death by 5-FU could contribute to its ability to prevent tumor-induced immunosuppression. However, in contrast to doxorubicin, 5-FU failed to induce calreticulin expression on EL4 tumor cells. ${ }^{[22]}$ Interestingly, 5-FU conserved its anticancer properties in TLR4-deficient tumor-bearing mice, further under- scoring that the anticancer activity of 5-FU is not related to the induction of immunogenic cell death. We also monitored the levels of Tregs in 5-FU-treated tumor-bearing mice. In contrast to cyclophosphamide administration that resulted in reduced frequency of splenic Tregs in vivo, 5-FU-treated tumor-bearing mice featured comparable Tregs levels to untreated controls, suggesting that 5-FU anticancer effects are driven by MDSC targeting. ${ }^{[22]}$ These findings are in line with the results indicating that the induction of Tregs from human PBMC is not affected by 5-FU. ${ }^{[25]}$ In vivo, elimination of MDSCs triggered by 5-FU promoted IFN $\gamma$ secretion by tumor-specific CD8 T cells. This was relevant as we noted that 5-FU was unable to control tumor progression in T-cell-deficient nude mice, underscoring that $\mathrm{T}$ cells are essential for the anticancer effects of 5-FU. ${ }^{[22]}$ Overall, our results suggest that the anticancer effects of 5-FU can be explained by its ability to target MDSCs and restore T cell anticancer immune responses.

\section{Therapeutic strategies enhancing in vivo anticancer effects of 5-FU}

Thus, 5-FU not only has the ability to induce direct cancer cell death but also can promote CD8 $\mathrm{T}$ cell activation and MDSC elimination. Nevertheless, despite its ability to trigger immunomodulatory effects in vivo, the anticancer effect of 5-FU remains limited. This has prompted scientists to combine 5-FU with other immunomodulating agents to enhance its anticancer effect in vivo. In a murine model of leukemia, Gol et al. have tested the efficacy of a combination of 5-FU with IL-12. ${ }^{[26]}$ Treatment of mice bearing L1210 leukemia with IL-12 or 5-FU alone triggered limited anticancer effects. Importantly, combined therapy with both agents markedly increased mouse survival and led to complete elimination of tumor cells in more than $70 \%$ of treated mice. ${ }^{[26]}$ The authors noted that the therapeutic efficacy of the combined therapy relied on T-cell-dependent adaptive immunity since the success of the combined treatment was absent in T-cell-deficient mice or mice treated with neutralizing antibodies against CD3-, CD4-, or CD8-positive cells. Upon testing the putative contribution of innate immune responses in the anticancer response mediated by $5-\mathrm{FU}$ and IL-12, the authors found that peritoneal macrophages, but not NK cells, were essential for the success of the combined therapy. ${ }^{[26]}$ The benefit of combining immunomodulatory cytokines with 5-FU has also been investigated in rats bearing transplantable colon carcinoma. IL-2 failed to significantly enhance 5-FU anticancer activity, but increased its toxicity. ${ }^{[27]}$ In contrast, combining IL-15 with 5-FU greatly enhanced its anticancer effect and limited 5-FU-driven gastrointestinal toxicity, ${ }^{[27]}$ thus supporting the combination of 5-FU and IL-15 [Figure 1]. 
Therapeutic strategies designed to trigger innate immune cell activation have also been tested in combination with 5-FU. Using an adenoviral vector driving the expression of mouse Flt3L, a cytokine that induces DC accumulation, Hou et al. found that adenoviral-mediated intratumoral expression of Flt3L combined with 5-FU therapy cured the established hepatomas and colon cancers in mice. Interestingly, such combination therapy elicited tumor-specific immunity and stimulated proliferation of lymphocytes, DCs, and NK cells. ${ }^{[28]}$ In an orthotopic model of pancreatic cancer, it was found that combining 5-FU with interferon-alpha (IFN $\alpha$ ) led to tumor cell infiltration by NK cells and resulted in enhanced 5-FU anticancer activity. This therapeutic effect was dependent on the presence of DCs, NK cells, and CD8 T cells. The combined treatment also augmented the expression of NKG2D ligands on cancer cells, possibly explaining enhanced NK cell cytotoxicity against cancer cells in this tumor model [Figure 1]. ${ }^{[29]}$ The potential of NK cells in promoting tumor regression in the context of 5-FU-based chemoimmunotherapy was further underscored by a recent study investigating the molecular bases explaining the efficacy of a combination therapy between 5-FU and $\alpha$-galactosylceramide ( $\alpha$-GalCer). Upon testing the efficacy of this combined therapy against liver tumors of MC38 colon cancer cells, Aketa et al. unraveled that $\alpha \mathrm{GalCer}$ induced liver NK cell activation while 5-FU augmented expression of NKG2D activating ligands on tumor cells. The combined action of these agents resulted in NK cell-dependent anticancer effects against tumor cells in vivo. ${ }^{[30]} \mathrm{NK}$ cells were similarly found to be critical mediators of the anticancer efficacy of a combined therapy using DC vaccine and 5-FU. ${ }^{[31]}$

5-FU-based combination therapies can also result in the activation of $\mathrm{T}$ cells that prevent cancer cell growth. Low-dose chemotherapy using 5-FU and cisplatin, followed by intratumoral injection of DCs was indeed found to drive CD8 T cell cytotoxicity, leading to the rejection of murine MC38 colon tumors. ${ }^{[32]}$ Correale et al. found that 5-FU was also able to enhance the ability of a peptide-based vaccine to induce anticancer $\mathrm{CD} 8 \mathrm{~T}$ cell responses in mice. ${ }^{[33]}$ Low-dose 5-FU administration combined with therapeutic adenoviral vaccination featured synergistic anticancer effects in tumor-bearing mice. 5-FU increased the levels of CD8-specific $\mathrm{T}$ cells in the spleens and draining lymph nodes of vaccinated mice. ${ }^{[34]}$ We also attempted to develop therapeutic strategies designed to augment the anticancer effect of 5-FU. Given that 5-FU reduces MDSC frequencies but fails to affect Treg cells, we tested the potential benefit of combining 5-FU with cyclophosphamide, a Treg depleting agent, to restore anticancer immune responses. We observed that this combination therapy was highly effective at controlling tumor growth in immunocompetent mice. ${ }^{[22]}$ Interestingly, T-cell-deficient nude mice were unresponsive to this combined therapy, underscoring the crucial role of
T-cell-dependent immune responses for the success of the combined therapy with cyclophosphamide and 5-FU. ${ }^{[22]}$ More recently, we gained further insight into the molecular immune mechanisms accounting for the anticancer effects of 5-FU. We observed that 5-FU induced early caspase-1 activation in MDSCs before triggering the death of MDSCs. This activation was dependent on the activation of NLRP3 inflammasome, a macromolecular complex involved in the activation of caspase-1. ${ }^{[11]}$ We searched for the molecular mechanisms accounting for the ability of 5-FU to activate NLRP3. Unlike classical NLRP3 activators that induce intracellular radical oxygen species (ROS) or potassium efflux, 5-FU triggers NLRP3 activation by driving lysosome permeabilization and the subsequent release of cathepsin $\mathrm{B}$. This lysosomal rupture was due to the ability of 5-FU to drive BAX activation. BAX indeed promotes apoptosis and mitochondrial and lysosomal permeabilization. Accordingly, genetic prevention of BAX expression in MDSCs prevented 5-FU-driven lysosome permeabilization. We further found that cathepsin B binds to NLRP3 and we speculate that this event induces a change in NLRP3 conformation that leads to its activation. It is noteworthy that caspase- 1 activation in MDSCs occurred $12 \mathrm{~h}$ after the administration of 5-FU. This was unexpected as this delay is longer compared to classical NLRP3 activators and is possibly due to the time required for 5-FU to affect nucleic acid synthesis in MDSCs. Whether caspase- 1 is also directly involved in the death of MDSCs upon 5-FU treatment remains to be determined.

Administration of 5-FU to tumor-bearing mice thus sets off a series of events that results in the secretion of low levels of IL- $1 \beta$ from MDSCs. We further found that MDSC-driven IL-1 $\beta$ release was responsible for the induction of IL-17secreting CD4 T cells (Th17 cells). Since IL-17a has been shown to promote tumor angiogenesis, ${ }^{[35,36]}$ we tested the efficacy of 5-FU in IL-17a-deficient mice and found that the absence of this cytokine reinforced the anticancer activity of 5-FU. ${ }^{[11]}$ Similarly, enhanced efficacy of 5-FU was noted in caspase-1-, NLRP3-, or IL-1R-deficient mice, underscoring that the NLRP3 $\rightarrow$ caspase- $1 \rightarrow$ IL-1 $\beta \rightarrow$ IL-1R1 $\rightarrow$ IL-17a signaling pathway compromises the effect of 5-FU in vivo. Accordingly, combined therapy using 5-FU with IL-1 receptor antagonist, which is used for the treatment of rheumatoid arthritis, ${ }^{[37]}$ boosted 5-FU chemotherapeutic activity. In metastatic colorectal cancer patients receiving 5-FU-based chemotherapy, we found that IL- $1 \beta$ serum concentrations were augmented in 9 out of 12 patients $24 \mathrm{~h}$ after 5 -FU treatment. In line with our studies on mouse, we also noted that 5-FU drove cathepsin B activity in MDSCs in most patients. ${ }^{[11]}$ In addition, we further found that 5-FU also drove caspase-1 activation in MDSC and augmented IL-17 secretion from PBMC. Collectively, these results indicate that 5-FU features ambivalent immunological effects. ${ }^{[38]}$ 5-FU kills MDSCs, thus restoring potent immune anticancer responses. How- 
ever, the 5-FU-driven caspase-1 activation in MDSCs leads to the promotion of tumor growth. While these results are clearly relevant for the design of future immunotherapeutic strategies using 5-FU, they might not apply to other classical chemotherapies used in a clinical setting. We had indeed previously reported that the anticancer effect of anthracyclines and oxaliplatin was dependent on the release of IL-1 $\beta$ from DCs and the subsequent activation of CD8 T-cell-dependent anticancer immunity. ${ }^{[7-9]}$ This means that depending on the environmental context, acute inflammation triggered by chemotherapy can actually be beneficial to the host. We think that our current observations with 5-FU do not contradict these previous findings because 5-FU does not actually restore CD8 T cell functions by inducing immunogenic tumor cell death, but rather by eliminating MDSCs. ${ }^{[1]} 5$-FU does not, therefore, activate CD8+ T cells through DC-mediated IL- $1 \beta$ release. Accordingly, our results indicate that MDSCs release only little IL-1 $\beta$ upon 5-FU treatment in contrast to DCs exposed to TLR4 inducers like HMGB1. Overall, these results are compatible with a scenario where high IL-1 $\beta$ concentrations favor CD8 T cell activation, but low IL-1 $\beta$ concentrations rather promote Th17 cell differentiation. Our mouse and human observations collectively provide strong impetus to combine 5-FU with immumomodulators aimed at blocking IL-1 and/or IL-17 activity. Based on our preclinical results, we have initiated a phase II clinical trial assessing the potential benefit of the addition of IL-1 receptor antagonist to 5-FU-based chemotherapy in metastatic colon cancer patients. This study will provide us with valuable information regarding the ability of 5-FU-based chemotherapy to enhance IL-17 production by human T cells and the impact of IL-1 blockade on IL-17-driven tumor angiogenesis in a clinical setting.

\section{Concluding remarks}

While 5-FU was originally designed to selectively prevent cancer cell proliferation, it has now become clear that this drug exhibits several immunomodulatory effects. Initial observations indicating that immune responses contributed to 5-FU anticancer effect have raised enthusiasm among scientists working in the field, but 5-FU given alone fails to eliminate established cancers, underscoring the need to combine 5-FU with other chemotherapeutic drugs and immunomodulators. Several studies have indeed shown that combinations of 5-FU with other chemotherapy drugs, cytokines, or DC vaccines could enhance 5-FU anticancer efficacy. To date, however, no study has assessed the therapeutic benefit of combining 5-FU with blockade of molecules negatively regulating $\mathrm{T}$ cell activation, such as Programmed cell death protein 1 ( PD-1), despite a report indicating that 5-FU drives human breast cancer cell resistance by promoting Programmed cell death 1 ligand 1 (PD-L1) surface expression in tumor cells. ${ }^{[39]}$ Whether the blockade of immune checkpoint molecules in addition to 5-FU administration can result in potent anticancer responses thus remains to be investigated [Figure 1]. Understanding the molecular mechanisms explaining the efficacy of combined 5-FU-based treatments will be essential to identify the biomarkers indicative of its therapeutic efficacy.

\section{Acknowledgements}

The authors are supported by grants from the Ligue Nationale contre le Cancer (F.G.), the Institut National du Cancer (F.G.), the Association pour la recherche sur le cancer (F.G. and L.A.), the Conseil Régional de Bourgogne (F.G. and L.A.), the FEDER (F.G. and L.A.), the Fondation de France (L.A.), the Agence Nationale de la Recherche [ANR-13-JSV3-0001-01] (L.A.) and [ANR-11-LABX-0021], the Ligue Régionale contre le cancer Comité Grand-Est (L.A.), and the European Community (Marie Curie Fellowship PCIG10-GA-2011-303719) (L.A.).

\section{REFERENCES}

1. Rutman RJ, CantarowA, Paschkis KE. Studies in 2-acetylaminofluorene carcinogenesis. III. The utilization of uracil-2-C14 by preneoplastic rat liver and rat hepatoma. Cancer Res 1954;14:119-23.

2. Heidelberger C, Chaudhuri NK, Danneberg P, Mooren D, Griesbach L, Duschinsky R, et al. Fluorinated pyrimidines, a new class of tumour-inhibitory compounds. Nature 1957;179:663-6.

3. Wohlhueter RM, McIvor RS, Plagemann PG. Facilitated transport of uracil and 5-fluorouracil, and permeation of orotic acid into cultured mammalian cells. J Cell Physiol 1980;104:309-19.

4. Falcone A, Ricci S, Brunetti I, Pfanner E, Allegrini G, Barbara C, et al. Phase III trial of infusional fluorouracil, leucovorin, oxaliplatin, and irinotecan (FOLFOXIRI) compared with infusional fluorouracil, leucovorin, and irinotecan (FOLFIRI) as first-line treatment for metastatic colorectal cancer: The Gruppo Oncologico Nord Ovest. J Clin Oncol 2007;25:1670-6.

5. Conroy T, Desseigne F, Ychou M, Bouche O, Guimbaud R, Becouarn $\mathrm{Y}$, et al. FOLFIRINOX versus gemcitabine for metastatic pancreatic cancer. N Engl J Med 2011;364:1817-25.

6. Obeid M, Tesniere A, Ghiringhelli F, Fimia GM, Apetoh L, Perfettini JL, et al. Calreticulin exposure dictates the immunogenicity of cancer cell death. Nat Med 2007;13:54-61.

7. Apetoh L, Ghiringhelli F, Tesniere A, Obeid M, Ortiz C, Criollo A, et al. Toll-like receptor 4-dependent contribution of the immune system to anticancer chemotherapy and radiotherapy. Nat Med 2007;13:1050-9.

8. Ghiringhelli F, Apetoh L, Tesniere A, Aymeric L, Ma Y, Ortiz C, et al. Activation of the NLRP3 inflammasome in dendritic cells induces IL-1beta-dependent adaptive immunity against tumors. Nat Med $2009 ; 15: 1170-8$

9. Apetoh L, Mignot G, Panaretakis T, Kroemer G, Zitvogel L. Immunogenicity of anthracyclines: Moving towards more personalized medicine. Trends Mol Med 2008;14:141-51.

10. Lake RA, Robinson BW. Immunotherapy and chemotherapy- a practical partnership. Nat Rev Cancer 2005;5:397-405. 
11. Bruchard M, Mignot G, Derangere V, Chalmin F, Chevriaux A, Vegran $\mathrm{F}$, et al. Chemotherapy-triggered cathepsin $\mathrm{B}$ release in myeloid-derived suppressor cells activates the NLRP3 inflammasome and promotes tumor growth. Nat Med 2013;19:57-64.

12. Ghiringhelli F, Apetoh L. The interplay between the immune system and chemotherapy: Emerging methods for optimizing therapy. Expert Rev Clin Immunol 2014;10:19-30.

13. Zitvogel L, Kepp O, Kroemer G. Immune parameters affecting the efficacy of chemotherapeutic regimens. Nat Rev Clin Oncol 2011;8:151-60.

14. Galetto A, Buttiglieri S, Forno S, Moro F, Mussa A, Matera L. Drug- and cell-mediated antitumor cytotoxicities modulate cross-presentation of tumor antigens by myeloid dendritic cells. Anticancer Drugs 2003;14:833-43.

15. Bergmann-Leitner ES, Abrams SI. Treatment of human colon carcinoma cell lines with anti-neoplastic agents enhances their lytic sensitivity to antigen-specific CD8+ cytotoxic T lymphocytes. Cancer Immunol Immunother 2001;50:445-5.

16. Backus HH, Dukers DF, van Groeningen CJ, Vos W, Bloemena E, Wouters D, et al. 5-Fluorouracil induced Fas upregulation associated with apoptosis in liver metastases of colorectal cancer patients. Ann Oncol 2001;12:209-16.

17. Eisenthal A, Eytan K, Brazowski E, Katz BZ, Shirazi I, Skornick Y. Effects of 5-fluorouracil on human mitogen-activated peripheral blood lymphocytes from healthy individuals. Anticancer Res 2008;28:3785-91.

18. Gabrilovich DI, Nagaraj S. Myeloid-derived suppressor cells as regulators of the immune system. Nat Rev Immunol 2009;9:162-74.

19. Zou W. Regulatory T cells, tumour immunity and immunotherapy. Nat Rev Immunol 2006;6:295-307.

20. Ghiringhelli F, Menard C, Puig PE, Ladoire S, Roux S, Martin F, et al. Metronomic cyclophosphamide regimen selectively depletes $\mathrm{CD} 4+\mathrm{CD} 25+$ regulatory $\mathrm{T}$ cells and restores $\mathrm{T}$ and $\mathrm{NK}$ effector functions in end stage cancer patients. Cancer Immunol Immunother 2007;56:641-8.

21. Suzuki E, Kapoor V, Jassar AS, Kaiser LR, Albelda SM. Gemcitabine selectively eliminates splenic Gr-1+/CD11b+myeloid suppressor cells in tumor-bearing animals and enhances antitumor immune activity. Clin Cancer Res 2005;11:6713-21.

22. Vincent J, Mignot G, Chalmin F, Ladoire S, Bruchard M, Chevriaux A, et al. 5-Fluorouracil selectively kills tumor-associated myeloid-derived suppressor cells resulting in enhanced $\mathrm{T}$ cell-dependent antitumor immunity. Cancer Res 2010;70:3052-61.

23. Qu X, Felder MA, Perez Horta Z, Sondel PM, Rakhmilevich AL. Antitumor effects of anti-CD40/CpG immunotherapy combined with gemcitabine or 5-fluorouracil chemotherapy in the B16 melanoma model. Int Immunopharmacol 2013;17:1141-7.

24. Kroemer G, Galluzzi L, Kepp O, Zitvogel L. Immunogenic cell death in cancer therapy. Annu Rev Immunol 2013;31:51-72.

25. Kan S, Hazama S, Maeda K, Inoue Y, Homma S, Koido S, et al. Suppressive effects of cyclophosphamide and gemcitabine on regulatory T-cell induction in vitro. Anticancer Res 2012;32:5363-9.
26. Gol b J, Zagozdzon R, Kaminski R, Kozar K, Gryska K, Izycki $\mathrm{D}$, et al. Potentiatied antitumor effectiveness of combined chemo-immunotherapy with interleukin-12 and 5-fluorouracil of L1210 leukemia in vivo. Leukemia 2001;15:613-20.

27. Cao S, Troutt AB, Rustum YM. Interleukin 15 protects against toxicity and potentiates antitumor activity of 5-fluorouracil alone and in combination with leucovorin in rats bearing colorectal cancer. Cancer Res 1998;58:1695-9.

28. Hou S, Kou G, Fan X, Wang H, Qian W, Zhang D, et al. Eradication of hepatoma and colon cancer in mice with Flt3L gene therapy in combination with 5-FU. Cancer Immunol Immunother 2007;56:1605-13.

29. Khallouf H, Marten A, Serba S, Teichgraber V, Buchler MW, Jager D, et al. 5-Fluorouracil and interferon-alpha immunochemotherapy enhances immunogenicity of murine pancreatic cancer through upregulation of NKG2D ligands and MHC class I. J Immunother 2012;35:245-53.

30. Aketa H, Tatsumi T, Kohga K, Tsunematsu H, Aono S, Shimizu S, et al. The combination therapy of alpha-galactosylceramide and 5 -fluorouracil showed antitumor effect synergistically against liver tumor in mice. Int J Cancer 2013;133:1126-34.

31. Nagasaki E, Takahara A, Koido S, Sagawa Y, Aiba K, Tajiri H, et al. Combined treatment with dendritic cells and 5-fluorouracil elicits augmented NK cell-mediated antitumor activity through the tumor necrosis factor-alpha pathway. J Immunother 2010;33:467-74.

32. Tanaka F, Yamaguchi H, Ohta M, Mashino K, Sonoda H, Sadanaga N, et al. Intratumoral injection of dendritic cells after treatment of anticancer drugs induces tumor-specific antitumor effect in vivo. Int J Cancer 2002;101:265-9.

33. Correale P, Del Vecchio MT, Di Genova G, Savellini GG, La Placa M, Terrosi C, et al. 5-fluorouracil-based chemotherapy enhances the antitumor activity of a thymidylate synthase-directed polyepitopic peptide vaccine. J Natl Cancer Inst 2005;97:1437-45.

34. Geary SM, Lemke CD, Lubaroff DM, Salem AK. The combination of a low-dose chemotherapeutic agent, 5-fluorouracil, and an adenoviral tumor vaccine has a synergistic benefit on survival in a tumor model system. PLoS One 2013;8:e67904.

35. Berger H, Vegran F, Chikh M, Gilardi F, Ladoire S, Bugaut H, et al. SOCS3 transactivation by PPARgamma prevents IL-17-driven cancer growth. Cancer Res 2013;73:3578-90.

36. Numasaki M, Fukushi J, Ono M, Narula SK, Zavodny PJ, Kudo T, et al. Interleukin-17 promotes angiogenesis and tumor growth. Blood 2003;101:2620-7.

37. Mertens M, Singh JA. Anakinra for rheumatoid arthritis. Cochrane Database Syst Rev 2009;1:CD005121.

38. Ghiringhelli F, Bruchard M, Apetoh L. Immune effects of 5-fluorouracil: Ambivalence matters. Oncoimmunology 2013;2:e23139.

39. Zhang P, Su DM, Liang M, Fu J. Chemopreventive agents induce programmed death-1-ligand 1 (PD-L1) surface expression in breast cancer cells and promote PD-L1-mediated T cell apoptosis. Mol Immunol 2008;45:1470-6. 